\title{
Metastatic Soft Tissue Tumors
}

\author{
Tomoaki Torigoe ${ }^{1}$, Atsuhiko Terakado ${ }^{1}$, Yoshiyuki Suehara ${ }^{1}$, Taketo Okubo ${ }^{1}$, Tatsuya Takagi ${ }^{1}$, \\ Kazuo Kaneko ${ }^{1}$, Yasuo Yazawa ${ }^{2}$
}

${ }^{1}$ Department of Orthopaedic Surgery, School of Medicine, Juntendo University, Tokyo, Japan; ${ }^{2}$ Department of Orthopaedic Oncology, Saitama International Medical Center, Saitama, Japan.

E-mail: ttorigoe@juntendo.ac.jp

Received October $15^{\text {th }}$, 2011; revised November $13^{\text {th }}$, 2011; accepted November 30 ${ }^{\text {th }}, 2011$.

\begin{abstract}
Between 1998 and 2007, we treated 157 patients with malignant soft tissue tumors. Patients with obvious lymph node metastasis, metastasis from bone or soft tissue sarcoma, and with obvious advanced stage disease which had multiple metastases were excluded from the present analysis. Finally, sixteen of the 157 patients were diagnosed to have soft tissue metastasis from carcinomas or hematopoietic malignancies. There were 10 males and 6 females with an average age of 65 (range, 50 - 83) years. Four lesions were located in the abdomen and back, 3 in the thigh, 2 in the chest, and 1 lesion each in the upper arm, lower leg and buttock. There were 9 intramuscular and 7 subcutaneous metastases. The average tumor size was $6 \mathrm{~cm}$ (range 2 - $17 \mathrm{~cm}$ ). The initial diagnoses were a primary soft tissue tumor in 8 cases, primary soft tissue tumor or metastatic soft tissue tumor in 6 , and inflammatory lesions in 2. Four of the 6 cases were suspected to be a metastatic soft tissue tumor in the initial diagnosis based on a past history of malignancy. There were no charac-teristic findings of metastatic soft tissue tumors in the imaging studies. The primary origins were lung cancer in 6 cases, malignant lymphoma in 5, gastric cancer in 2, and esophageal cancer, pancreatic cancer and uterine cancer in 1 patient each. Treatments were administered to all cases, including radiotherapy and chemotherapy in 11, chemotherapy in 2, wide resection and chemotherapy in 1, radiotherapy in 1 and palliative therapy in 1 . The final oncological status was continuous disease free survival in 1 patient, no evidence of disease in 1, alive with disease in 7 , and dead of disease in 7 patients. The estimated one-year survival was $47 \%$ and the two-year survival was $0 \%$.
\end{abstract}

Keywords: Soft Tissue Tumor, Metastasis, Carcinoma, Lymphoma, Inflammation

\section{Introduction}

Metastatic soft tissue tumors from malignant tumors are considered to be rare compared to metastatic bone tumors. The presence of a soft tissue mass caused by a metastasis could be misdiagnosed as a primary soft tissue sarcoma or inflammatory lesion based on the physical findings and imaging studies. The clinical distinction between a metastatic soft tissue tumor and primary soft tissue sarcoma and inflammation is important, because the treatment and prognosis are markedly different. Although soft tissue sarcomas are often cured by wide resection and/or adjuvant chemotherapy and radiotherapy, metastatic soft tumors are mainly treated with chemotherapy and radiotherapy, and the prognosis is poor. Moreover, if a metastatic soft tumor is misdiagnosed as an inflammatory lesion, conservative treatment with antiinflammatory medication may be prescribed, thereby possibly delaying appropriate treatment.

\section{Patients and Methods}

We retrospectively reviewed 157 patients with soft tissue malignant tumors treated at Juntendo University Hospital and Juntendo Orthopedic Oncology Collaboration (JOOC) affiliated hospitals between 1998 and 2007. The clinical and radiological records of all 157 patients were reviewed. A histopathologocal diagnosis was obtained for all of the cases. Patients with lymph node metastasis, soft tissue metastasis from bone, and soft tissue sarcoma were excluded from the study. However, multiple soft tissue metastases were observed occasionally in patients with advanced carcinoma, and such cases were also excluded in this study. Because our aim was to discriminate between primary soft tissue sarcoma and metastatic soft tissue tumors, making a diagnosis was not difficult in advanced cases. The survival rates were estimated using the Kaplan-Meier method.

\section{Results}

There were 10 males and 6 females with metastatic soft tissue tumors arising from other malignant tumors. The ages of the patients at diagnosis ranges from 50 to 83 years (average 65). Their chief complains were a soft 
tissue mass with or without pain in all cases. Four cases had a past history of carcinoma. A biopsy was performed in all cases, in 13 it was a needle biopsy, and in three an open biopsy was performed. The location of the metastasis was the abdomen in four cases, back in four, thigh in three, chest in two, and upper arm, lower leg and buttock in one case each. There were nine intramuscular lesions and seven subcutaneous lesions. The tumor size ranged from 2 to $17 \mathrm{~cm}$ (average $6 \mathrm{~cm}$ ).

The initial diagnosis was a primary soft tissue tumor in eight cases, primary soft tissue tumor or metastatic soft tissue tumor in four cases, and primary soft tissue tumor or lymphoma in two cases. All of the four cases suspected to be metastatic soft tissue tumors had a past history of malignancy. Two cases were initially diagnosed as inflammatory lesions, and were subsequently diagnosed as metastases from lung cancer of adenocarcinoma histology. The primary site was the lungs in six cases, lymphoma in five cases, gastric cancer in two cases, and the esophagus, pancreas cancer and uterus in one case each (Table 1).

In imaging studies, CT and MRI were effective for detecting for soft tissue tumors, but there were no characteristic findings of metastatic soft tissue tumors, and the discrimination between primary soft tissue sarcoma and metastatic soft tissue tumor was difficult using these modalities (Figures 1(a) and (b)). Gallium scintigraphy is beneficial for making a diagnosis of malignant lymphoma, but it less effective for diagnosing other malignancies. Positron emission tomography (PET) was effective for detecting soft tissue tumors, particularly for the detection of multiple lesions, but there were also no characteristic findings of metastatic soft tissue tumors by PET (Figure 2).

Table 1. Summary of 16 patients with metastatic soft tissue tumor.

\begin{tabular}{|c|c|c|c|c|c|c|c|c|c|c|}
\hline Case & Age/Sex & Location & Muscle/Subcutaneous & $\begin{array}{l}\text { size } \\
(\mathrm{cm})\end{array}$ & $\begin{array}{c}\text { Initial } \\
\text { Diagnosis }\end{array}$ & $\begin{array}{c}\text { Primary } \\
\text { Malignancy }\end{array}$ & $\begin{array}{c}\text { Previous } \\
\text { Malignancy }\end{array}$ & Treatment & $\begin{array}{l}\text { Final } \\
\text { Status }\end{array}$ & $\begin{array}{l}\text { Follow-up } \\
\text { (M) }\end{array}$ \\
\hline 1 & $66 / F$ & Back & M & 3 & PSTT & Lymphoma & None & $\mathrm{CTx}+\mathrm{RTx}$ & DOD & 20 \\
\hline 2 & $79 / \mathrm{M}$ & Upper arm & $\mathrm{S}$ & 2 & Inflammation & Lung & None & Palliative & AWD & 4 \\
\hline 3 & 79/M & Back & M & 10 & PSTT & Lung & None & CTx + RTx & DOD & 4 \\
\hline 4 & $68 / \mathrm{M}$ & Abdomen & $\mathrm{S}$ & 3 & PSTT & Lymphoma & None & $\mathrm{CTx}+\mathrm{RTx}$ & $\mathrm{CDF}$ & 10 \\
\hline 5 & $67 / \mathrm{M}$ & Lower leg & M & 2 & PSTT/MSTT & Esophagus & Esophagus & $\mathrm{CTx}+\mathrm{RTx}$ & DOD & 4 \\
\hline 6 & $78 / \mathrm{M}$ & Thigh & M & 4 & PSTT/MSTT & Lung & Lung & $\mathrm{CTx}+\mathrm{RTx}$ & AWD & 12 \\
\hline 7 & $60 / \mathrm{F}$ & Back & M & 5 & PSTT & Lung & None & $\mathrm{CTx}+\mathrm{RTx}$ & DOD & 7 \\
\hline 8 & $60 / \mathrm{M}$ & Back & M & 3 & PSTT/MSTT & Lung & Lung & WR + CTx & NED & 4 \\
\hline 9 & $58 / \mathrm{M}$ & Buttock & M & 17 & PSTT/Lymphoma & Lymphoma & None & CTx + RTx & AWD & 7 \\
\hline 10 & $71 / \mathrm{M}$ & Chest & $\mathrm{S}$ & 3 & PSTT & Lymphoma & None & $\mathrm{CTx}+\mathrm{RTx}$ & AWD & 4 \\
\hline 11 & $50 / \mathrm{F}$ & Abdomen & M & 4 & PSTT/MSTT & Stomach & Stomach & CTx + RTx & DOD & 6 \\
\hline 12 & $53 / \mathrm{M}$ & Thigh & M & 4 & Inflammation & Lung & None & $\mathrm{CTx}+\mathrm{RTx}$ & AWD & 7 \\
\hline 13 & $69 / F$ & Chest & $\mathrm{S}$ & 15 & PSTT/Lymphoma & Lymphoma & None & CTx & AWD & 1 \\
\hline 15 & $42 / \mathrm{F}$ & Abdomen & $\mathrm{S}$ & 3 & PSTT & Pancreas & None & CTx + RTx & DOD & 4 \\
\hline 16 & 83/M & Thigh & $\mathrm{S}$ & 3 & PSTT & Stomach & None & $\mathrm{RTx}$ & DOD & 10 \\
\hline
\end{tabular}

M, Muscle; S, Subcutaneous; PSTT, Primary Soft Tissue Tumor; MSTT, Metastatic Soft Tissue Tumor; CTx, Chemotherapy; RTx, Radiotheraty; WR, Wide Resection; CDF, Continuous Disease Free Survival; NED, No Evidence of Disease; AWD, Alive with Disease; DOD, Dead of Disease. 


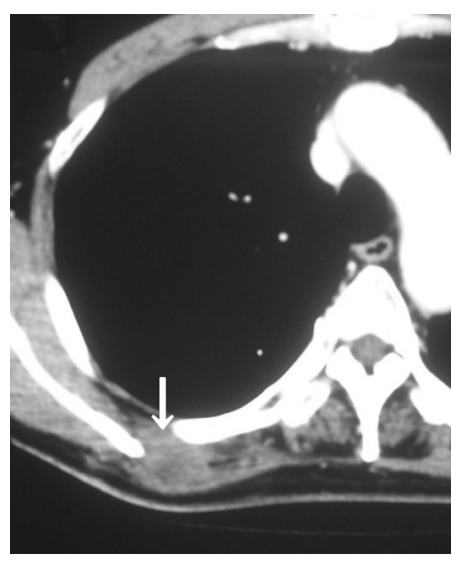

(a)

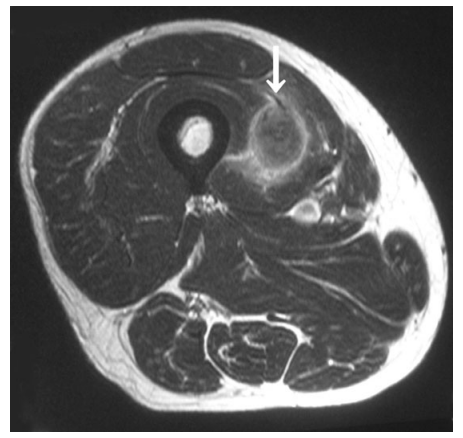

(b)

Figure 1. The results of the imaging studies of metastatic soft tissue tumors. (a) CT revealed a low enhanced lesion in the rhomboid muscle. This lesion was a skeletal muscle metastasis from lung cancer; (b) MRI revealed a heterogeneous high signal intense lesion in the quadriceps muscle on axial T2-weighted images.

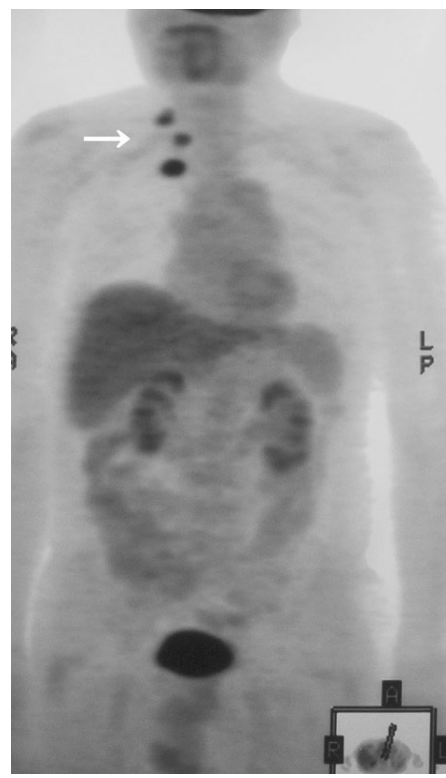

Figure 2. PET revealed three round lesions on the back. These were skeletal muscle metastasis from lung cancer.
Three cases were initially diagnosed as inflammatory lesions. One case was a 79 year-old male, who reported pain and swelling in his left elbow without any identified episodes for a few months. The pain and swelling in the elbow were progressive, and he was referred to our hospital. No mass lesion was palpable in the left elbow during the first visit. The patient had a high fever and no past history of any malignant tumors. MRI revealed an iso- to slightly high signal intense lesion on T1-weighted images, and heterogeneous high signal intensity lesion on T2 images of the subcutaneous tissue in the left elbow. The border of the lesion was indistinct. MRI showed diffuse high intensity changes around the soft tissue of the lesion on T2 and fat-suppressed Gadolinium enhanced studies, and these findings were considered to indicate inflammation (Figures 3(a)-(c)). Chest, abdominal and pelvic CT studies were performed to identify the cause of the patient's fever. Lesions were detected in the left lung and right iliopsoas muscle, and these lesions were also considered to be due to inflammation (Figures 4(a) and (b)).

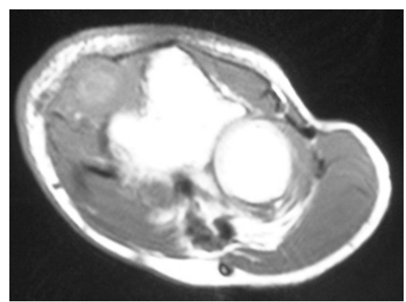

(a)

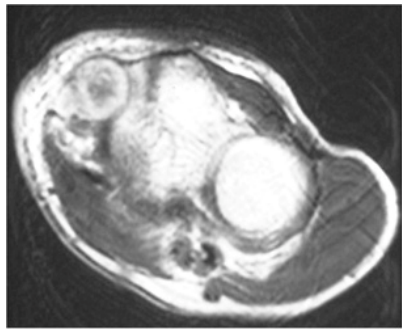

(b)

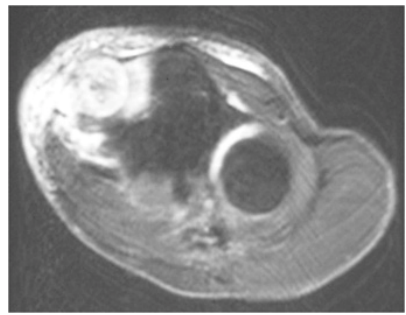

(c)

Figure 3. (a) MRI revealed an iso- to slightly high signal intense lesion in the subcutaneous tissue in the left elbow on axial T1-weighted images; (b) MRI revealed a heterogeneous high signal intensity lesion on T2; (c) MRI revealed diffuse high intensity changes around the soft tissue of the lesion on an axial fat-suppressed Gadolinium enhanced study. 
In laboratory studies, a hematological examination showed increased white blood cell and platelet counts. The chemical analysis showed an increase in the CRPlevel. The tumor markers were all within the normal limits except for the soluble interleukin 2 receptor. Our clinical diagnosis was a multifocal abscess. We treated the patient with antibiotics, but his clinical findings did not improve. We therefore suspected a neoplastic condition, and a needle biopsy from the elbow lesion was performed. Histopathological specimens showed tumor cells with large nucleoli, and the tumor cells had a ductual structure (Figure 5). In the immunochemical study, cytokeratin was positive in the tumor cells, so the pathological diagnosis was a metastasis of the soft tissue from adenocarcinoma. The final diagnosis was lung cancer with soft tissue metastasis.

The other case was initially diagnosed with an inflammatory lesion in the muscle based on the findings of an MRI study. The initial diagnosis was sarcoidosis, but lung cancer was detected in the chest CT which was performed during an examination for bilateral hilar lymphadenopathy of sarcoidosis.

As treatment for the metastatic soft tissue tumors, radiotherapy and chemotherapy were performed in 11 cases. Only chemotherapy was administered in two cases. Radiotherapy, and wide resection combined with chemotherapy, were performed in one case each. One patient

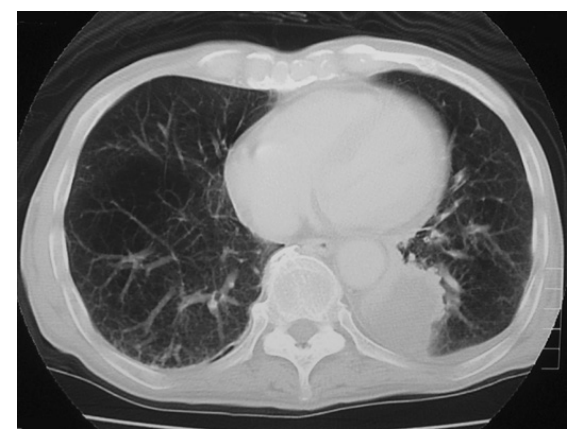

(a)

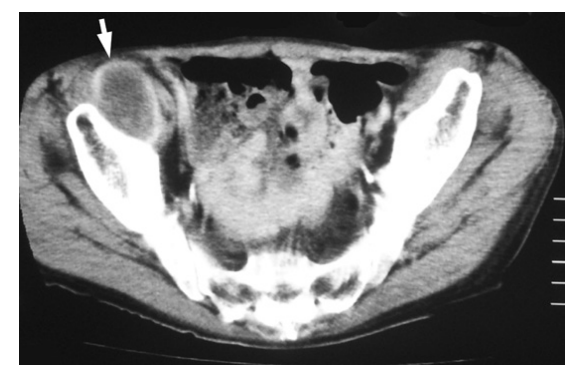

(b)

Figure 4. (a) Plain CT revealed a lesion with ill-defined margins in the left lung; (b) Enhanced CT revealed a round lesion in the right iliopsoas muscle, and only the border of the lesion showed contrast enhancement.

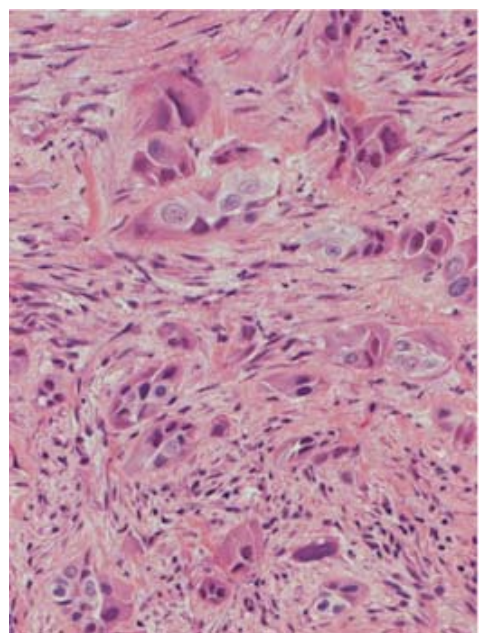

Figure 5. The histopathological specimens showed tumor cells with large nucleoli, and the tumor cells had a ductal structure.

was treated with palliative care only because the patient was of an advanced age and thus was deemed to be unable to withstand the strain of other treatments.

The final status of the patients was continuous disease free survival in one case, no evidence of disease in one, alive with disease in seven, and dead of disease in seven cases. The estimated 1-year survival rate was $47 \%$, and the 2 -year survival rate was $0 \%$.

\section{Discussion}

Distant metastasis to soft tissue has rarely been reported in the literature [1,2]. Willis reported a frequency of $0.8 \%$, and Pearson reported a frequency of $5.5 \%$ from autopsy studies $[3,4]$. Autopsies do not generally include a check for soft tissue masses, so these data may not have been accurate. However, Tuoheti reported a frequency of $0.2 \%$ and Sudo reported a frequency of $2.7 \%$ based on clinical studies $[5,6]$. Therefore there are major differences in the reported frequency of metastatic soft tissue tumors, and these differences are considered to have de-pended on the procedures used for the examinations. Although the number of cases which had soft tissue metastasis as the first symptom of disease was low, the number of subjects with soft tissue metastasis increased when more frequent examinations of cancer patients were performed, and when autopsy cases were examined.

With regard to the metastatic site, Damron reported that metastases were identified in the thigh in $17 \%$, back in $13 \%$, chest in $10 \%$, upper arm in $10 \%$ and lower leg in $7 \%$ of their cases. Plaza reported that the tumors were located in the abdominal wall in $21 \%$, back in $17 \%$, thigh in $14 \%$, chest wall in $13 \%$ and arm in $13 \%$ of their cases $[7,8]$. In our series, metastases were identified in the abdominal wall in $25 \%$, back in $25 \%$, thigh in $19 \%$, chest 
wall in $13 \%$, and arm in $6 \%$. These findings were considered to showing a similar tendency with regard to the tumor location.

As the primary malignant tumor, Plaza reported that the most common source was skin cancer in $16 \%$ of their subjects, followed by cancers of the lung $11 \%$, breast $11 \%$, kidney $10 \%$ and colon $10 \%$. Damron reported that the most frequent tumor of origin was lung cancer in $35 \%$ of their patients, followed by the kidney in $13 \%$, and colon in $8 \%[7,9]$. In our series, the primary malignancies were lung cancer in 38\%, lymphoma in 31\%, stomach cancer in $13 \%$, and esophageal, pancreatic and uterine cancer in $6 \%$ of patients, each. Although our series showed different tendencies from the literature, we considered that differences in ethnicity and the different definitions of metastatic soft tissue tumors between our study and those studies were likely responsible for these differences. In our series, lymphoma was relatively common as the source of the metastasis, while Masaoka reported that the frequency of malignant lymphoma in patient with soft tissue metastases was low, which was similar to other reports [10]. The specific influence of our institution and the numerous patients with hemato-poietic tumors was considered to be the cause of this difference.

Regarding the initial diagnoses of the soft tissue metastatic tumor, Damron reported that the diagnosis was primary soft tissue sarcoma in $43 \%$, and an abscess and cyst in 7\% each, and that an unknown was considered for $37 \%$ of the cases [8]. In our series, the diagnosis as a primary soft tissue tumor was made in $50 \%$ of cases. A primary soft tissue tumor or metastatic soft tissue tumor was diagnosed in $25 \%$ of cases. There were no cases which could be initially diagnosed as a metastatic soft tissue tumor without a past history of previous malignancy, because the metastatic soft tissue tumors did not show any characteristic findings in the imaging studies, so it was difficult to differentiate soft tissue metastasis from soft tissue sarcoma. Confirmation of a past history was considered to be important for the early diagnosis.

Moreover abscess and inflammation were considered to be important differential diagnoses of soft tissue metastasis form cancers. Because the MRI findings of soft tissue inflammation often include nonspecific low signal intensity on T1-weighted images and high signal intensity on T2-weighted images due to significant edema, it is sometimes similar to metastatic soft tissue tumors [11]. In our series, three of the 17 patients (18\%) were initially misdiagnosed to have inflammatory lesions. Williams reported that abscesses and hematoma were included as the differential diagnoses of soft tissue metastasis by MRI findings in their study [12].

The treatment used for the metastatic soft tissue tumors was mainly radiation and chemotherapy in our se- ries. Single treatment or combination treatment including wide resection were also performed. Damron reported that combination treatment with radiation and chemotherapy was indicated for metastatic soft tissue tumors in $40 \%$ of cases, radiation alone in $24 \%$, radiation and resection in $20 \%$, radiation and resection and chemotherapy in 13\%, and no treatment in 3\%. He reported 1 and 2 year survival rates of $25 \%$ and $12 \%$ [7]. Our estimated 1 and 2 year survival rates were $47 \%$ and $0 \%$, so the prognosis of soft tissue tumor was considered to be poor. There was no tendency for a surgery for the metastatic lesion to improve the prognosis of the metastatic soft tissue tumors, similar to other malignant tumors with distant metastasis, and the indications for surgery for metastatic soft tumors are considered to be limited to the cases in which radiation therapy can not provide local control.

\section{REFERENCES}

[1] K. S. Sridhar, R. K. Rao and B. Kunhardt, "Skeletal Muscle Metastases from Lung Cancer,” Cancer, Vol. 59, No. 8, 1987, pp. 1530-1534. doi:10.1002/1097-0142(19870415)59:8<1530::AID-CNC R2820590824>3.0.CO;2-H

[2] P. P. McKeown, P. Conant and L. E. Auerbach, "Squamous Cell Carcinoma of the Lung: An Unusual Metastasis to Pectoralis Muscle," The Annals of Thoracic Surgery, Vol. 61, No. 5, 1996, pp. 1525-1526. doi:10.1016/0003-4975(95)01177-3

[3] R. A. Willis, "The Spread of Tumours in the Human Body,” Butterworth and Co., London, 1952.

[4] C. M. Pearson, "Incidence and Type of Pathologic Alterations Observed in Muscle in a Routine Autopsy Survey," Neurology, Vol. 9, 1959, pp. 757-766.

[5] Y. Tuoheti, K. Okada, T. Osanai, J. Nishida, S. Ehara, M. Hashimoto and E. Itoi, "Skeletal Muscle Metastases of Carcinoma: A Clinicopathological Study of 12 Cases," Japanese Journal of Clinical Oncology, Vol. 34, No. 3, 2004, pp. 210-214. doi:10.1093/jico/hyh036

[6] A. Sudo, Y. Ogihara, Y. Shiokawa, S. Fujinami and S. Sekiguchi, "Intramuscular Metastasis of Carcinoma," Clinical Orthopaedics and Related Research, Vol. 296, 1993, pp. 213-217

[7] T. A. Damron and J. Heiner, "Distant Soft Tissue Metastases: A Series of 30 New Patients and 91 Cases from the Literature,” Annals of Surgical Oncology, Vol. 7, No. 7, 2000, pp. 526-534. doi:10.1007/s10434-000-0526-7

[8] J. A. Plaza, D. Perez-Montiel, J. Mayerson, C. Morrison and S. Suster, "Metastases to Soft Tissue: A Review of 118 Cases over a 30-Year Period," Cancer, Vol.112, No. 1, 2008, pp. 193-203. doi:10.1002/cncr.23151

[9] T. A. Damron and J. Heiner, "Management of Metastatic Disease to Soft Tissue," Orthopedic Clinics of North America, Vol. 31, No. 4, 2000, pp. 661-673. 


\section{doi:10.1016/S0030-5898(05)70183-8}

[10] S. Masaoka and T. Fu, "Malignant Lymphoma in Skeletal Muscle with rhabdomyolysis: A Report of Two Cases," Journal of Orthopaedic Science, Vol. 7, No. 6, 2002, pp. 688-693. doi:10.1007/s007760200122

[11] J. B. Williams, R. A. Youngberg, L. T. Bui-Mansfield and J. D. Pitcher, "MR Imaging of Skeletal Muscle Metastases,” American Journal of Roentgenology, Vol. 168, No. 2, 1997, pp. 555-557.

[12] G. R. Avery, "Metastatic Adenocarcinoma Masquerading as a Psoas Abscess," Clinical Radiology, Vol. 39, No. 3, 1988, pp. 319-320. doi:10.1016/S0009-9260(88)80554-3 